\title{
Anti-Cancer Effects of Green Tea by Either Anti- or Pro- Oxidative Mechanisms
}

\author{
Sumio Hayakawa ${ }^{1 *}$, Kieko Saito ${ }^{2}$, Noriyuki Miyoshi ${ }^{3}$, Tomokazu Ohishi ${ }^{4}$, Yumiko \\ Oishi $^{1}$, Mamoru Miyoshi ${ }^{2}$, Yoriyuki Nakamura ${ }^{2}$
}

\begin{abstract}
Tea derived from the leaves and buds of Camellia sinensis (Theaceae) is consumed worldwide. Green tea contains various components with specific health-promoting effects, and is believed to exert protective effects against diseases including cancer, diabetes and hepatitis, as well as obesity. Of the various tea components, the polyphenol catechins have been the subject of extensive investigation and among the catechins, (-)-epigallocatechin gallate has the strongest bioactivity in most cases. Our research group has postulated that hepatocyte nuclear factor-4 $\alpha$, sterol regulatory element-binding proteins, and tumor necrosis factor- $\alpha$ are targets of green tea constituents including (-)-epigallocatechin gallate for their anti-diabetes, anti-obesity, and anti-hepatitis effects, respectively. Published papers were reviewed to determine whether the observed changes in these factors can be correlated with anti-cancer effects of green tea. Two major action mechanisms of (-)-epigallocatechin gallate have been proposed; one associated with its anti-oxidative properties and the other with its pro-oxidative activity. When reactive oxygen species are assumed to be involved, our findings that (-)-epigallocatechin gallate downregulated hepatocyte nuclear factor-4 $\alpha$, sterol regulatory element-binding proteins, and tumor necrosis factor- $\alpha$ may explain the anti-cancer effect of green tea as well. However, further studies are required to elucidate which determinant directs (-)-epigallocatechin gallate action as an anti-oxidant or a pro-oxidant for favorable activity.
\end{abstract}

Keywords: Green tea - catechin - epigallocatechin gallate - cancer - reactive oxygen species

Asian Pac J Cancer Prev, 17 (4), 1649-1654

\section{Introduction}

Tea is produced from the leaves and buds of the Camellia sinensis (Theaceae) plant, and is one of the world's most popular beverages. Tea can be broadly classified according to the production method as green tea, oolong tea, black tea, or pu-erh tea. Green tea is mainly consumed in Japan and China, whereas black tea is primarily consumed in Western countries, India, and other parts of the world.

Green tea was first brought to Japan, more than 1000 years ago, from China as a form of medicine. In 1211, the Japanese Zen monk Eisai published a book entitled "Kissa Youjouki" which means "promotion of health by tea", and described that "tea is a marvelous preventive medicine to maintain people's health and has an extraordinary power to prolong life" (Miyoshi et al., 2015). Indeed, recent scientific evidence has shown that green tea is beneficial for health-promotion (Yang et al., 2009; Khan and Mukhtar, 2010; Singh et al., 2011; Yang and Wang, 2011; Suzuki et al., 2012; Khan and Mukhtar, 2013; Miyoshi et al., 2015; Yang et al., 2016).
Green tea and tea polyphenol called catechins are believed to exert protective effects against diseases such as cancer, obesity, diabetes, arteriosclerosis, neurodegenerative diseases, tooth decay, hepatitis, allergy, and bacterial and viral infection (Yang et al., 2009; Khan and Mukhtar, 2010; Singh et al., 2011; Yang and Wang, 2011; Suzuki et al., 2012; Khan and Mukhtar, 2013; Miyoshi et al., 2015; Yang et al., 2016). Among the green tea catechins (GTCs), (-)-epigallocatechin gallate (EGCG, Figure 1) exhibits the strongest bioactivity in general,<smiles>O=C(O[C@H]1Cc2c(O)cc(O)cc2O[C@H]1c1cc(O)c(O)c(O)c1)c1cc(O)c(O)c(O)c1</smiles>

Figure 1. Chemical Structure of (-)-Epigallocatechin Gallate (EGCG)

${ }^{1}$ Department of Cellular and Molecular Medicine, Medical Research Institute, Tokyo Medical and Dental University, Tokyo, ${ }^{2}$ Tea Science Center, ${ }^{3}$ Graduate School of Integrated Pharmaceutical and Nutritional Sciences, Graduate Program in Food and Nutritional Sciences, University of Shizuoka, ${ }^{4}$ Institute of Microbial Chemistry (BIKAKEN), Shizuoka, Japan *For correspondence: hayakawa. dcmm@mri.tmd.ac.jp 
and EGCG is almost exclusively contained in $C$. sinensis species (Miyoshi et al., 2015). Majority of scientific evidence based on cellular and animal experiments has indicated that green tea can exert beneficial health effects on cancer, although the evidence relating to its effects in humans remains inconclusive (Yang et al., 2009; Singh et al., 2011; Yang and Wang, 2011; Suzuki et al., 2012; Khan and Mukhtar, 2013; Miyoshi et al., 2015).

Our research group has demonstrated several lines of evidence to show the green tea's actions against cancer, hepatitis, and diabetes. We postulated that hepatocyte nuclear factor (HNF)- $4 \alpha$, sterol regulatory elementbinding proteins (SREBPs), and tumor necrosis factor (TNF)- $\alpha$ are the targets of green tea constituents including EGCG in anti-diabetes, anti-obesity, and anti-hepatitis effects, respectively (Suzuki et al., 2012). However, it is not clear whether or not the observed changes in these factors can also be correlated with the anti-cancer effect of green tea.

In this review, we discuss how these factors can be linked to the green tea's anti-cancer action, which would provide an example to interconnect various findings on mechanisms proposed for green tea's preventive actions against various diseases.

\section{Effects of green tea on $\mathrm{HNF}-4 \alpha$}

One of the hallmarks of diabetes is the failure to control the blood glucose level (Waltner-Law et al., 2002). The postprandial glucose level is maintained by gluconeogenesis in which gluconeogenic enzymes, phosphoenolpyruvate carboxykinase and glucose-6phosphatase are involved. EGCG was demonstrated to inhibit glucose production in hepatoma cells and primary hepatocytes by reducing the gene and protein expression of these enzymes (Waltner-Law et al., 2002; Collins et al., 2007). Our animal experiment also revealed that green tea and EGCG reduced their gene expressions in the liver (Koyama et al., 2004). Later, we found that inhibition of HNF-4 $\alpha$ was correlated with these findings (Yasui et al., 2010; Suzuki et al., 2012; Miyoshi et al., 2015). This transcription factor binds to the promoter to regulate the gene expression of phosphoenolpyruvate carboxykinase and glucose-6-phosphatase (Suzuki et al., 2012). Therefore, green tea consumption is expected to contribute to the reduction in the postprandial blood glucose level, suggesting its favorable effect in diabetes.

Several human studies have shown the beneficial effect of green tea consumption on diabetes (Yang et al., 2009; Singh et al., 2011; Yang and Wang, 2011; Suzuki et al., 2012; Khan and Mukhtar, 2013; Miyoshi et al., 2015; Yang et al., 2016). For example, a large scale retrospective cohort study found the inverse association between consumption of green tea and the risk of diabetes (Iso et al., 2006), and an intervention study revealed that the blood glucose level increased by oral glucose burden was mitigated by consumption of green tea (Tsuneki et al., 2004). Bogdanski et al. found that long term supplementation of green tea extract (GTE) caused the reduction in the fasting serum glucose level in obese, hypertensive subjects (Bogdanski et al., 2012).

\section{Effects of green tea on SREBPs}

SREBPs are the transcription factors which play a central role in regulation of cellular lipogenesis and lipid homeostasis (Weber et al., 2004). Several studies have shown that the fat-lowering effect of green tea and EGCG is mediated by SREBPs (Shrestha et al., 2009; Santamarina et al., 2015). In diet-induced obese mice, dietary EGCG lowered the levels of plasma triglycerides and liver lipid (Lee et al., 2009). In the epididymal white adipose tissue, EGCG decreased the mRNA levels of adipogenic genes such as SREBP-1c, peroxisome proliferator-activated receptor- $\gamma$, and fatty acid synthase. Our animal experiment showed that oral administration of a catechin-free fraction reduced plasma levels of cholesterol and triglycerides, with concomitant reduction in hepatic gene expression of SREBPs (Suzuki et al., 2012; Yasui et al., 2012; Miyoshi et al., 2015). Thus, green tea contains lipid-lowering components in addition to GTC.

Several human studies have shown that green tea and EGCG have the lipid lowering effect (Wang et al., 2014). For example, in a double-blind, placebo-controlled trial on 56 obese, hypertensive subjects consuming a daily supplement of 1 capsule with 379 mg of GTE for 3 months, the GTE group was found to show reductions in the total and low-density lipoprotein cholesterol and triglycerides as compared with the placebo group (Bogdanski et al., 2012).

\section{Effects of green tea on TNF- $\alpha$}

TNF is a proinflammatory cytokine. Fujiki and his coworkers have demonstrated that the anti-cancer effect of green tea can be correlated with inhibitory effect on gene and protein expression of TNF- $\alpha$ (Suganuma et al., 2000).

Our animal experiment using galactosamine-induced hepatitis model rats showed the consumption of green tea beverage with a high content of EGCG attenuated hepatitis (Abe et al., 2005; Suzuki et al., 2012; Miyoshi et al., 2015). The effect was correlated with the beverage's inhibition of the gene and protein expression of inflammatory cytokines, TNF- $\alpha$ and interleukin $1 \beta$. Similarly, EGCG reduced the mRNA levels of TNF- $\alpha$ and interleukin- $1 \beta$ in the mesenteric adipose tissue of non-obese type 2 diabetic rats when they were given a diet containing $0.1 \%$ EGCG for 25 weeks (Uchiyama et al., 2013). These finding are compatible with the result of a human clinical study demonstrating that GTE caused reduction in serum levels of TNF- $\alpha$ and C-reactive protein (Bogdanski et al., 2012).

\section{Anti-cancer effects of green tea}

Most of cellular and animal experiments have demonstrated the anti-cancer effects of green tea and EGCG (Yang et al., 2009; Khan and Mukhtar, 2010; Singh et al., 2011; Yang and Wang, 2011; Suzuki et al., 2012; Khan and Mukhtar, 2013; Miyoshi et al., 2015). The major compound contributing to the activity of green tea is believed to be EGCG.

Human epidemiological and intervention studies have also shown that green tea and tea catechins exert preventive 
effects against various cancers (Yang et al., 2009; Singh et al., 2011; Yang and Wang, 2011; Suzuki et al., 2012; Khan and Mukhtar, 2013; Miyoshi et al., 2015). Of 127 case-control studies and 90 cohort studies published from 1965 to 2008,51 case-control studies and 19 cohort studies showed an inverse association between green/ black tea consumption and cancer risk for various types of cancer including colon, lung, stomach, breast, prostate, ovarian, pancreatic, kidney, and bladder cancers (Yang et al., 2009) and a growing body of evidence supports green tea's favorable effects (Miyoshi et al., 2015).

On the other hand, many findings have failed to demonstrate such effects. These conflicting results may arise from several confounding factors, including the methods of quantifying tea consumption, tea temperature, cigarette smoking, alcohol consumption, and the differences in genetic and environmental factors such as race, sex, age, and lifestyle (Yang et al., 2009; Yang and Wang, 2011; Suzuki et al., 2012; Miyoshi et al., 2015). The different results between human and animal experiments could have been due to the different doses of tea (Khan and Mukhtar, 2013).

\section{Anti-cancer mechanism of green tea through anti-oxidant activity (Figure 2)}

It is well established that GTCs such as EGCG have a strong anti-oxidant property (Yang et al., 2009; Khan and Mukhtar, 2010; Singh et al., 2011; Yang and Wang, 2011; Suzuki et al., 2012; Khan and Mukhtar, 2013; Miyoshi et al., 2015; Yang et al., 2016). For example, an intervention study with obese, hypertensive participants found that supplementation of GTE increased anti-oxidant status (Bogdanski et al., 2012). Chen et al. (2011) demonstrated that the elevated level of reactive oxygen species (ROS) in the liver of female rats treated with ethanol was attenuated by intravenous supplementation with GTCs.

Carcinogenic chemicals, cigarette smoke, radiation, and microorganisms are known to cause the generation of ROS to induce carcinogenesis (Lin et al., 1995; Aw, 1999; Li et al., 2003). ROS increase the expression and/ or activation of $\mathrm{NF}-\varkappa \mathrm{B}$ to stimulate the expression/activity of several cancer-related factors such as TNF- $\alpha$, nitric oxide, cyclooxygenase (COX)-2, matrix metalloproteinase (MMP)-9, and Bcl-2 (Lin et al., 1995; Sharma and Tepas, 2010; Choudhari et al., 2013; Lee and Yang, 2013).

TNF- $\alpha$ has a tumor-promotion effect and EGCG inhibited BALB/3T3 cell growth through inhibition of okadaic acid-mediated NF- $x \mathrm{~B}$ activation, being accompanied by inhibition of TNF-alpha gene expression (Suganuma et al., 2000). EGCG showed anti-cancer effect by inhibiting nitric oxide production through suppressing inducible nitric oxide synthase activity (Punathil et al., 2008). EGCG inhibited mammary cancer cell migration by suppressing the elevated levels of endogenous nitric oxide/nitric oxide synthase in 4T1 cells (Beltz et al., 2006). GTE was shown to down-regulate COX-2 induced by 12-O-tetradecanoylphorbol-13-acetate (TPA) in mouse skin and in TPA-stimulated human mammary cells, suggesting its anti-cancer effect (Singh et al., 2011). We observed that EGCG inhibited growth of fibrosarcoma
Carcinogenic chemicals, radiation, microorganisms

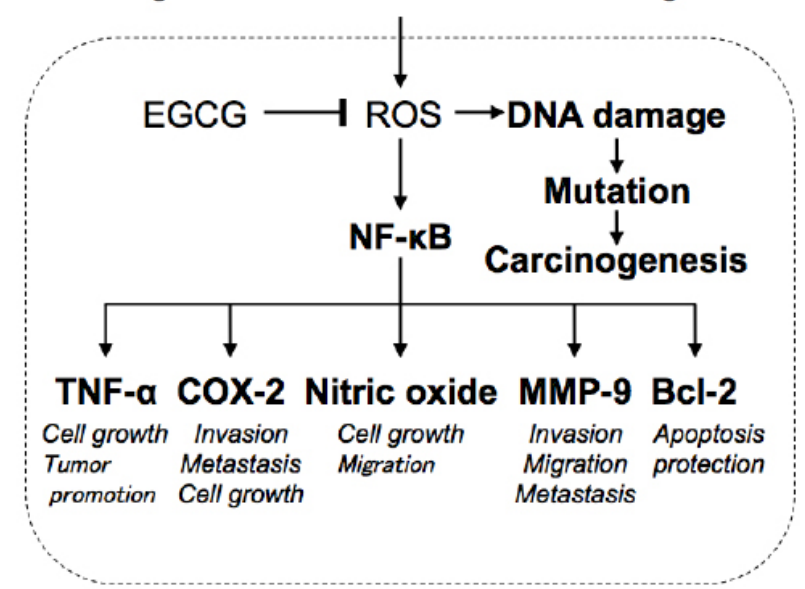

Figure 2. Mechanism of Action of EGCG as an Antioxidant. ROS cause DNA damage, which leads to carcinogenesis, and activate $\mathrm{NF}-x \mathrm{~B}$, which promotes generation of various factors including TNF- $\alpha$, COX-2, nitric oxide, MMP-9, and Bcl-2. The anti-oxidative effect of EGCG reduces ROS production, which leads to its anti-cancer effect.

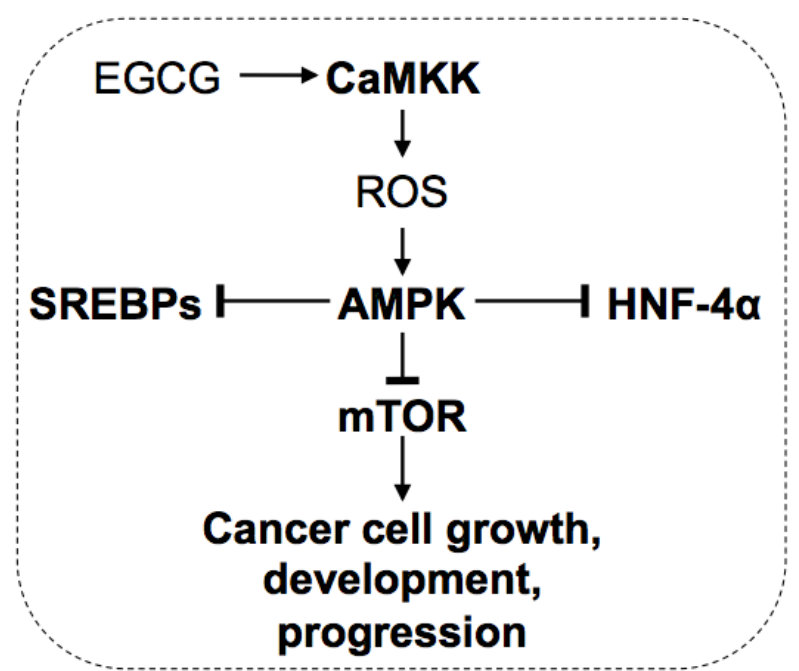

Figure 3. Mechanism of Action of EGCG as a Prooxidant. EGCG stimulates the production of ROS, which promotes AMPK activation, leading to inhibition of the expressions of SREBPs, HNF- $4 \alpha$, and mTOR, which play the crucial roles in cancer growth, development, progression, and metastasis. Thus, EGCG exhibits the anti-cancer effect by reducing the level of these cancer-promoting proteins.

HT1080 cells together with down-regulation of MMP9 expression (Maeda-Yamamoto et al., 2003). EGCG induced apoptosis in HT-1080 cells by the mechanism in which suppression of anti-apoptotic protein $\mathrm{Bcl}-2$ expression and NF- $x \mathrm{~B}$ activation are involved through its anti-oxidant activity (Lee et al., 2011).

\section{Anti-cancer mechanism of green tea through the pro-oxidant activity (Figure 3 )}

Several lines of evidence have indicated the prooxidant activity of EGCG (Yang et al., 2009; Singh et al., 2011; Yang and Wang, 2011; Suzuki et al., 2012). We found that EGCG induced oxidative stress to lead to apoptosis in cultured tumor cells (Saeki et al., 2002). EGCG may 
increase the ROS generation through the action on $\mathrm{Ca}^{2+}$ / calmodulin-dependent protein kinase kinase (Collins et al., 2007). ROS can activate 5'-AMP-activated protein kinase (AMPK) (Collins et al., 2007; Hwang et al., 2007; Babu et al., 2013), and the activated AMPK may inhibit the expression of HNF-4 $\alpha$ (Schwartz et al., 2009; Steinberg and Kemp, 2009), SREBP (Motoshima et al., 2006; Steinberg and Kemp, 2009; Liu et al., 2015), COX-2 (Hwang et al., 2007), and mammalian target of rapamycin (mTOR) which up-regulates cell profiferation (Motoshima et al., 2006). Therefore, these transcription-regulating factors can be the targets of anti-cancer drugs.

Schwartz et al. (Schwartz et al., 2009) examined the effect of HNF- $4 \alpha$ antagonists and HNF-4 $\alpha$ siRNA on growth and proliferation of colorectal cancer cells in xenotransplanted nude mice. The results indicated that these agents inhibited growth and proliferation of HT29 and Caco 2 cells by a mechanism which involves activation of caspase-3 and down-regulation of Bcl-2 leading to apoptotic death. Animal experiments gave similar results. Thus, the tumor suppression was accounted for by suppression of HNF-4 $\alpha$ transcription and protein expression. These authors have proposed that HNF- $4 \alpha$ is a target for an anti-cancer drug.

Hwang et al. (Hwang et al., 2007) demonstrated that EGCG treatment of colon cancer cells resulted in a strong activation of AMPK in association with an inhibition of COX-2 expression. The decreased COX-2 expression as well as prostaglandin E2 secretion stimulated by EGCG was abolished by an AMPK inhibitor, Compound C, suggesting that AMPK can be a target for an anti-cancer strategy.

Accelerated synthesis of lipids such as fatty acid and cholesterol is a requirement for tumor cells to grow fast due to the obligatory requirement to constitute their cell membrane (Gabitova et al., 2014). SREBPs play the central role in the lipid metabolism and have been shown to induce cancer cell growth, development, progression and metastasis (Huang et al., 2012; Guo et al., 2014; Li et al., 2014a; Li et al., 2014b; Li et al., 2016). The high level of SREBP-1 protein expression was correlated with high mortality in patients with hepatocellular carcinoma and knockdown of SREBP-1 inhibited migration and invasion of hepatoma cells (Li et al., 2014a). Accordingly, blocking SREBPs can be a pharmacological intervention for cancer therapy (Li et al., 2014b). Thus, ROS generation by action of EGCG as a pro-oxidant contributes to down-regulation of SREBPs through AMPK activation, and consequently leads to the anti-cancer effect of green tea.

mTOR is involved in translocation of SREBPs to the Golgi, in which SREBPs are cleaved by protease and the fragment enters into the nucleus to promote lipid synthesis (Gabitova et al., 2014). Therefore, the pathway of phosphoinositide-3-kinase/protein kinase B/mTOR plays an important role in solid malignancies and inhibitors to the pathway are expected as the cancer therapeutic agent (Motoshima et al., 2006; Jokinen and Koivunen, 2015). Since AMPK negatively regulates mTOR expression (Motoshima et al., 2006), EGCG may exert its anti-cancer activity by stimulating the generation of ROS which activates AMPK to lead to the inhibition of mTOR.
Yang et al. have already pointed out the activation of AMPK is a common mechanism among various biological effects of EGCG (Yang et al., 2016).

\section{Concluding Remarks}

The anti-cancer effect of green tea can be explained by either its anti-oxidative or pro-oxidative property. As exemplified by ascorbate, EGCG can act either as an anti-oxidant or pro-oxidant depending on the cellular environment (Sakagami and Satoh, 1997; Waltner-Law et al., 2002; Park et al., 2006). The effect of green tea and EGCG may be different by the difference in a redox homeostasis threshold of different cells such as normal versus cancer cells (Kanadzu et al., 2006; Li et al., 2015). The tissue specific differences in membrane structure and metabolism may also contribute to the different effect.

In the experiment using diabetes model rats, consumption of a diet containing $0.1 \%$ EGCG reduced the oxidative stress, but not in 0.2 or $0.5 \%$ diet groups, suggesting the available concentration is one of the determinants for EGCG to be oxidative or anti-oxidative (Uchiyama et al., 2013). Beltz et al. described that at high concentrations, polyphenols such as EGCG have prooxidative activities, whereas at much lower levels, antioxidative effects occurred (Punathil et al., 2008). Further studies are required to understand what is a mechanism for EGCG to act as an anti-oxidant (Figure 2) or pro-oxidant (Figure 3), through which EGCG can exert its favorable activity on a disease.

In addition to the action on ROS, EGCG can modulate biological events through its protein-binding activity. Our research group demonstrated that EGCG directly bound to plasma proteins such as fibronectin and fibrinogen using EGCG immobilized on agarose gel (Sazuka et al., 1996). Since then a number of studies have demonstrated such direct binding in the effects of EGCG including anticancer effects (Yang et al., 2009; Singh et al., 2011; Yang and Wang, 2011; Suzuki et al., 2012; Miyoshi et al., 2015). Also, the experiment using a surface plasmon resonance assay led to the discovery of $67 \mathrm{kDa}$ laminin receptor as a cell surface receptor of EGCG in various activities including anti-cancer effect (Tachibana, 2011). Therefore, it is tempting to discuss whether or not a mechanism based on the EGCG's direct binding property by which green tea gives a favorable effect on a certain diseases can also be applied to other diseases, and this issue will be discussed elsewhere.

\section{References}

Abe K, Ijiri M, Suzuki T, et al (2005). Green tea with a high catechin content suppresses inflammatory cytokine expression in the galactosamine-injured rat liver. Biomed Res, 26, 187-92.

Aw TY (1999). Molecular and cellular responses to oxidative stress and changes in oxidation-reduction imbalance in the intestine. Am J Clin Nutr, 70, 557-65.

Babu PV, Liu D, Gilbert ER (2013). Recent advances in understanding the anti-diabetic actions of dietary flavonoids. J Nutr Biochem, 24, 1777-89.

Beltz LA, Bayer DK, Moss AL, et al (2006). Mechanisms of 
cancer prevention by green and black tea polyphenols. Anticancer Agents Med Chem, 6, 389-406.

Bogdanski P, Suliburska J, Szulinska M, et al (2012). Green tea extract reduces blood pressure, inflammatory biomarkers, and oxidative stress and improves parameters associated with insulin resistance in obese, hypertensive patients. Nutr Res, 32, 421-7.

Chen KH, Li PC, Lin WH, et al (2011). Depression by a green tea extract of alcohol-induced oxidative stress and lipogenesis in rat liver. Biosci Biotechnol Biochem, 75, 1668-76.

Choudhari SK, Chaudhary M, Bagde S, et al (2013). Nitric oxide and cancer: a review. World J Surg Oncol, 11, 118.

Collins QF, Liu HY, Pi J, et al (2007). Epigallocatechin-3gallate (EGCG), a green tea polyphenol, suppresses hepatic gluconeogenesis through 5'-AMP-activated protein kinase. J Biol Chem, 282, 30143-9.

Gabitova L, Gorin A, Astsaturov I (2014). Molecular pathways: sterols and receptor signaling in cancer. Clin Cancer Res, 20, 28-34.

Guo D, Bell EH, Mischel P, et al (2014). Targeting SREBP-1driven lipid metabolism to treat cancer. Curr Pharm Des, 20, 2619-26.

Huang WC, Li X, Liu J, et al (2012). Activation of androgen receptor, lipogenesis, and oxidative stress converged by SREBP-1 is responsible for regulating growth and progression of prostate cancer cells. Mol Cancer Res, 10, $133-42$.

Hwang JT, Ha J, Park IJ, et al (2007). Apoptotic effect of EGCG in HT-29 colon cancer cells via AMPK signal pathway. Cancer Lett, 247, 115-21.

Iso H, Date C, Wakai K, et al (2006). The relationship between green tea and total caffeine intake and risk for self-reported type 2 diabetes among Japanese adults. Ann Intern Med, 144, 554-62.

Jokinen E, Koivunen JP (2015). MEK and PI3K inhibition in solid tumors: rationale and evidence to date. Ther Adv Med Oncol, 7, 170-80.

Kanadzu M, Lu Y, Morimoto K (2006). Dual function of (--)-epigallocatechin gallate (EGCG) in healthy human lymphocytes. Cancer Lett, 241, 250-5.

Khan N, Mukhtar H (2010). Cancer and metastasis: prevention and treatment by green tea. Cancer Metastasis Rev, 29, 435-45.

Khan N, Mukhtar H (2013). Tea and health: studies in humans. Curr Pharm Des, 19, 6141-7.

Koyama Y, Abe K, Sano Y, et al (2004). Effects of green tea on gene expression of hepatic gluconeogenic enzymes in vivo. Planta Med, 70, 1100-2.

Lee IT, Yang CM (2013). Inflammatory signalings involved in airway and pulmonary diseases. Mediators Inflamm, 2013, 791231.

Lee MH, Han DW, Hyon SH, et al (2011). Apoptosis of human fibrosarcoma HT-1080 cells by epigallocatechin-3-O-gallate via induction of $\mathrm{p} 53$ and caspases as well as suppression of Bcl-2 and phosphorylated nuclear factor-kappaB. Apoptosis, 16, 75-85.

Lee MS, Kim CT, Kim Y (2009). Green tea (-)-epigallocatechin3 -gallate reduces body weight with regulation of multiple genes expression in adipose tissue of diet-induced obese mice. Ann Nutr Metab, 54, 151-7.

Li C, Yang W, Zhang J, et al (2014). SREBP-1 has a prognostic role and contributes to invasion and metastasis in human hepatocellular carcinoma. Int J Mol Sci, 15, 7124-38.

Li L, Steinauer KK, Dirks AJ, et al (2003). Radiation-induced cyclooxygenase 2 up-regulation is dependent on redox status in prostate cancer cells. Radiat Res, 160, 617-21.

Li P, Wu M, Wang J, et al (2015). NAC selectively inhibit cancer telomerase activity: A higher redox homeostasis threshold exists in cancer cells. Redox Biol, 8, 91-7.

Li X, Chen YT, Hu P, et al (2014b). Fatostatin displays high antitumor activity in prostate cancer by blocking SREBPregulated metabolic pathways and androgen receptor signaling. Mol Cancer Ther, 13, 855-66.

Li X, Wu JB, Li Q, et al (2016). SREBP-2 promotes stem celllike properties and metastasis by transcriptional activation of c-Myc in prostate cancer. Oncotarget, 7, 12869-84.

Lin KI, Lee SH, Narayanan R, et al (1995). Thiol agents and Bcl-2 identify an alphavirus-induced apoptotic pathway that requires activation of the transcription factor NF-kappa B. J Cell Biol, 131, 1149-61.

Liu HW, Chan YC, Wang MF, et al (2015). Dietary (-)-epigallocatechin-3-gallate supplementation counteracts aging-associated skeletal muscle insulin resistance and fatty liver in senescence-accelerated mouse. J Agric Food Chem, 63, 8407-17.

Maeda-Yamamoto M, Suzuki N, Sawai Y, et al (2003). Association of suppression of extracellular signal-regulated kinase phosphorylation by epigallocatechin gallate with the reduction of matrix metalloproteinase activities in human fibrosarcoma HT1080 cells. J Agric Food Chem, 51, 1858-63.

Miyoshi N, Pervin M, Suzuki T, et al (2015). Green tea catechins for well-being and therapy: prospects and opportunities. Botanics: Targets Therapy, 5, 85-96.

Motoshima H, Goldstein BJ, Igata M, et al (2006). AMPK and cell proliferation--AMPK as a therapeutic target for atherosclerosis and cancer. $J$ Physiol, 574, 63-71.

Park IJ, Hwang JT, Kim YM, et al (2006). Differential modulation of AMPK signaling pathways by low or high levels of exogenous reactive oxygen species in colon cancer cells. Ann N Y Acad Sci, 1091, 102-9.

Punathil T, Tollefsbol TO, Katiyar SK (2008). EGCG inhibits mammary cancer cell migration through inhibition of nitric oxide synthase and guanylate cyclase. Biochem Biophys Res Commun, 375, 162-7.

Saeki K, Kobayashi N, Inazawa Y, et al (2002). Oxidationtriggered c-Jun N-terminal kinase (JNK) and p38 mitogenactivated protein (MAP) kinase pathways for apoptosis in human leukaemic cells stimulated by epigallocatechin-3gallate (EGCG): a distinct pathway from those of chemically induced and receptor-mediated apoptosis. Biochem J, $\mathbf{3 6 8}$, 705-20.

Sakagami H, Satoh K (1997). Prooxidant action of two antioxidants: ascorbic acid and gallic acid. Anticancer Res, 17, 221-4.

Santamarina AB, Oliveira JL, Silva FP, et al (2015). Green Tea Extract Rich in Epigallocatechin-3-Gallate Prevents Fatty Liver by AMPK Activation via LKB1 in Mice Fed a HighFat Diet. PLoS One, 10, 141227.

Sazuka M, Itoi T, Suzuki Y, et al (1996). Evidence for the interaction between (-)-epigallocatechin gallate and human plasma proteins fibronectin, fibrinogen, and histidine-rich glycoprotein. Biosci Biotechnol Biochem, 60, 1317-9.

Schwartz B, Algamas-Dimantov A, Hertz R, et al (2009). Inhibition of colorectal cancer by targeting hepatocyte nuclear factor-4alpha. Int J Cancer, 124, 1081-9.

Sharma R, Tepas JJ, $3^{\text {rd }}$ (2010). Microecology, intestinal epithelial barrier and necrotizing enterocolitis. Pediatr Surg Int , 26, 11-21.

Shrestha S, Ehlers SJ, Lee JY, et al (2009). Dietary green tea extract lowers plasma and hepatic triglycerides and decreases the expression of sterol regulatory element-binding protein-1c mRNA and its responsive genes in fructose-fed, ovariectomized rats. J Nutr, 139, 640-5. 
Singh BN, Shankar S, Srivastava RK (2011). Green tea catechin, epigallocatechin-3-gallate (EGCG): mechanisms, perspectives and clinical applications. Biochem Pharmacol, 82, 1807-21.

Steinberg GR, Kemp BE (2009). AMPK in Health and Disease. Physiol Rev, 89, 1025-78.

Suganuma M, Sueoka E, Sueoka N, et al (2000). Mechanisms of cancer prevention by tea polyphenols based on inhibition of TNF-alpha expression. Biofactors, 13, 67-72.

Suzuki Y, Miyoshi N, Miyoshi M (2012). Health-promoting effects of green tea. Proc Jpn Acad Ser B Phys Biol Sci, 88, 88-101.

Tachibana H (2011). Green tea polyphenol sensing. Proc Jpn Acad Ser B Phys Biol Sci, 87, 66-80.

Tsuneki H, Ishizuka M, Terasawa M, et al (2004). Effect of green tea on blood glucose levels and serum proteomic patterns in diabetic $(\mathrm{db} / \mathrm{db})$ mice and on glucose metabolism in healthy humans. BMC Pharmacol, 4, 18.

Uchiyama Y, Suzuki T, Mochizuki K, et al (2013). Dietary supplementation with (-)-epigallocatechin-3-gallate reduces inflammatory response in adipose tissue of non-obese type 2 diabetic Goto-Kakizaki (GK) rats. J Agric Food Chem, 61, 11410-7.

Waltner-Law ME, Wang XL, Law BK, et al (2002). Epigallocatechin gallate, a constituent of green tea, represses hepatic glucose production. J Biol Chem, 277, 34933-40.

Wang S, Moustaid-Moussa N, Chen L, et al (2014). Novel insights of dietary polyphenols and obesity. J Nutr Biochem, 25, 1-18.

Weber LW, Boll M, Stampfl A (2004). Maintaining cholesterol homeostasis: sterol regulatory element-binding proteins. World J Gastroenterol, 10, 3081-7.

Yang CS, Wang H (2011). Mechanistic issues concerning cancer prevention by tea catechins. Mol Nutr Food Res, 55, 819-31.

Yang CS, Wang X, Lu G, et al (2009). Cancer prevention by tea: animal studies, molecular mechanisms and human relevance. Nat Rev Cancer, 9, 429-39.

Yang CS, Zhang J, Zhang L, et al (2016). Mechanisms of body weight reduction and metabolic syndrome alleviation by tea. Mol Nutr Food Res, 60, 160-74.

Yasui K, Paeng N, Miyoshi N, et al (2012). Effects of a catechinfree fraction derived from green tea on gene expression of enzymes related to lipid metabolism in the mouse liver. Biomed Res, 33, 9-13.

Yasui K, Tanabe H, Okada N, et al (2010). Effects of catechinrich green tea on gene expression of gluconeogenic enzymes in rat hepatoma H4IIE cells. Biomed Res, 31, 183-9. 